\title{
Risky Choices in Medical Decision Making: un Commentaire
}

\author{
par J.C. Sailly*
}

\section{Introduction}

Ayant lu avec grand intérêt la communication du Professeur Weinstein sur la prise de décision médicale dans un monde incertain, il m'est apparu que les commentaires ${ }^{1}$ d'un économiste pouvaient porter utilement sur deux types de réflexion. Tout d'abord, quel peut être l'apport de la démarche économique dans l'aide à la prise de décision médicale, y compris en incorporant la dimension éthique que celle-ci comporte? En second lieu, certains systèmes de santé favorisent-ils, davantage que d'autres, l'intégration de la perspective économique dans la prise de décision médicale?

\section{L'apport de la démarche économique dans l'aide à la prise de décision médicale}

Tous ne s'accordent pas à reconnaître que l'évolution importante du progrès médical et des nouvelles technologies ne permettent pas (en particulier depuis le resserrement des contraintes financières que nous connaissons de façon aiguë depuis maintenant une décennie) de faire bénéficier tout patient éventuel de toutes les investigations dont on dispose désormais [1]. Cette attitude conduit logiquement à refuser la prise en compte de la composante économique dans la prise de décision médicale, en particulier au nom de considérations éthiques. Une telle attitude nous apparaît fondée sur une conception erronée, et de la prise de décision médicale dans un contexte de ressources limitées, et de l'apport possible de la démarche économique dans cette prise de décision.

Aussi, avant d'examiner le degré de pertinence de cet apport, il peut être utile de rapporter et de critiquer quelques opinions émises parfois par certaines fractions du corps médical et qui tendent à nier ou à réduire fortement cet apport.

* Directeur du Centre de Recherches Economiques, Sociologiques et de Gestion, Lille (France). Chargé de recherche au CNRS.

${ }^{1}$ Ces commentaires doivent beaucoup aux remarques et suggestions de Thérèse LEBRUN, Chargée de Recherche INSERM au CRESGE, et Louis EECKHOUDT, professeur aux Facultés Catholiques de Lille et de Mons (B). 
2.1. Nous nous bornerons à relever quatre catégories d'assertions que, par faute de temps, nous livrons sans nuance [2] et [3].

Première assertion: «la santé est essentiellement une affaire médicale; son maintien ou sa restauration dépend donc du corps médical et, en conséquence, les décisions en ce domaine lui reviennent quasi exclusivement».

Or, on sait que le logement, l'alimentation, les conditions de travail, la qualité du réseau routier, l'hygiène de vie sont autant de facteurs qui entrent fortement en jeu dans le maintien de l'état de santé d'une population. Pour l'économiste, le problème suivant se pose donc: dans quel type d'action (y compris en dehors du secteur des soins de santé) faut-il mettre des moyens pour obtenir le maximum de surcroît de santé?

Deuxième assertion: "le milieu médical n'a pas à prendre en compte des éléments financiers dans sa décision: d'ailleurs ne dit-on pas que la santé n'a pas de prix?»

S'il est vrai que «la santé n'a pas de prix», il n'empêche, cependant, "qu'elle a un coût» [5] et qu'il n'est pas possible, que ce soit d'ailleurs au niveau individuel ou collectif, de lui consacrer la totalité des ressources dont on dispose. Dès lors, il faut bien effectuer des choix.

Troisième assertion: «tout médecin doit faire le maximum pour chaque malade pris isolément et ceci, au.nom même de l'éthique. En conséquence, puisqu'il faut tout faire, le problème des choix se pose avec moins d'acuité».

Cette position, qui est tout à fait à l'honneur du monde médical lorsqu'on la prend dans le sens commun (faire le maximum pour sauver une vie), peut devenir a-rationnelle et même antiéthique si l'on en fait la base d'un comportement qui se veut responsable.

En effet, les ressources du médecin sont limitées, que ce soient les thérapeutiques, les appareillages, etc..., qui sont à sa disposition, ou même le temps qu'il peut consacrer à son travail. Et donc, faire le maximum pour un malade, c'est priver d'autres malades des ressources ainsi utilisées pour soigner le premier. Le problème du médecin est bien de répartir au mieux les ressources rares que sont son temps et les sommes allouées à la santé par la collectivité. Cette allocation doit se faire entre plusieurs activités possibles (activités de recherche, activités de formation, activités de dépistage et prévention, activités de soins), et donc entre plusieurs malades possibles (les malades d'aujourd'hui et les malades de demain, les divers malades d'aujourd'hui, etc...). Choisir une activité parmi plusieurs, c'est exclure celles qui n'ont pas été retenues. Choisir de soigner tel malade, c'est refuser à un autre le bénéfice des moyens qui ont été octroyés au premier.

Le penchant du médecin est parfois d'optimiser les soins et la survie alors que le problème qui se pose à lui est d'optimiser le rapport coût-efficacité de sa décision. Autrement dit, le bénéfice de la solution retenue (et qui profite à tel patient) doit être comparé au sacrifice des solutions exclues (et qui pèsent sur d'autres patients). Une décision médicale ne prenant en considération que les seuls bénéfices pourrait se révéler contraire à l'éthique si ces bénéfices étaient inférieurs aux sacrifices entraînés par cette même décision.

Quatrième assertion: «le rôle des économistes (que l'on a tendance à confondre avec les comptables!) est de calculer les flux financiers engendrés par l'activité médicale et de les gérer au mieux». Dans une telle conception, la démarche économique relève moins d'une approche de type scientifique que d'une technique. Elle doit donc se contenter de mesurer des flux monétaires et n'a pas à interférer avec la décision médicale. A fortiori n'a-t-elle pas à être prise en considération par le médecin confronté à son choix. 
D'ailleurs, l'insistance des économistes à vouloir intervenir dans le cadre d'une rationalisation de cette décision peut apparaître tout à fait déplacée: n'est-il pas malséant d'essayer de mesurer en unités monétaires ce qui touche à un bien aussi précieux que la santé?

Cette quatrième assertion nous semble méconnaître l'originalité et l'intérêt de l'approche économique dans l'aide à la rationalisation des choix en matière de santé. C'est ce que nous voudrions montrer maintenant.

2.2 Rappelons tout d'abord que, dans toute problématique de prise de décision, deux aspects fondamentaux doivent être considérés:

- quels sont le volume et la diversité des éléments que l'on prend effectivement en compte dans le choix? Pour reprendre quelques-uns des éléments évoqués précédemment et à titre d'exemple, lorsqu'il s'agit de développer la santé, prend-on en considération les seules actions de soins curatives ou inclut-on dans le raisonnement l'amélioration des conditions de travail et la lutte contre le tabagisme? Pour les seules actions curatives, raisonne-t-on en fonction du seul malade que l'on a devant soi ou étend-on l'analyse aux autres malades pouvant bénéficier de ces actions? Même lorsque l'on s'intéresse à un seul malade, tient-on compte de l'estimation des avantages et inconvénients d'une décision par le seul médecin ou va-t-on jusqu'à intégrer l'estimation par le patient de ces mêmes paramètres?... [1] et [6] à [10].

On le voit, plus le nombre d'éléments incorporés dans l'analyse est grand, plus la diversité de points de vue introduite est large, plus la décision prise peut être pertinente, y compris au regard de considérations éthiques.

- Comment évaluer les différents coûts et bénéfices des éléments que l'on a ainsi décidé de prendre en compte en vue d'en arriver à une décision optimale? Quel étalon de mesure adopter pour effectuer cette opération?

La démarche économique apparaît particulièrement adaptée pour aider à résoudre, au moins partiellement, ce type de problèmes, et ceci, à quatre titres.

Tout d'abord, ayant pour objet de rechercher la combinaison optimale de ressources limitées, elle constitue, par essence, une méthode de choix tenant compte et des bénéfices et des coûts.

En second lieu, le type de raisonnement induit par cette démarche s'applique, par nature, à la considération d'un champ très vaste d'utilisation de moyens rares: c'est ainsi que l'on peut essayer de déterminer quelle est l'efficience la meilleure, en terme de niveau de santé atteint, d'un franc supplémentaire consacré à diverses actions possibles, ces actions pouvant aller de l'aménagement d'un carrefour dangereux, d'une campagne d'information sanitaire, à l'accentuation de l'effort de recherche ou au remboursement d'un nouvel examen complémentaire.

En troisième lieu, l'analyse économique, depuis les origines de son élaboration en tant que science, a tenté, d'une part, de tenir compte des niveaux d'analyse micro et macroéconomique, d'autre part, d'intégrer le temps dans ses modèles. Or, on a vu plus haut combien ces prises en considération étaient particulièrement nécessaires dans l'étude des divers effets de décisions médicales [14]. C'est le cas du niveau collectif ou macroéconomique dans l'intégration des aspects de santé publique ou de limitation des ressources financières, techniques et humaines pour la collectivité; c'est le cas du niveau microéconomique dans l'aide au diagnostic médical et dans l'analyse des préférences du patient [11] et [12]. C'est enfin le cas de l'introduction du temps dans la prise de décision, notamment dans le domaine de l'évaluation des technologies ou de nouvelles procédures [13]. 
En dernier lieu, l'analyse économique propose un moyen, parmi d'autres, de valoriser, et donc, d'homogénéiser, différents points de vue, en particulier par l'étalon monétaire.

Loin de prétendre tout ramener à des valeurs monétaires, elle offre, au contraire, une méthodologie permettant de prendre en compte une multitude de points de vue et d'améliorer la mesure comparée de leurs avantages et de leurs inconvénients.

La place de l'économiste aux côtés du médecins n'est pas donc pas le reflet d'une hégémonie déplacée. Elle se traduit au contraire par un apport particulièrement adapté à la nature des problèmes rencontrés dans la décision médicale: Prise en compte simultanée des atteintes, des performances techniques des appareillages et procédures disponibles, des thérapeutiques, des préférences des patients, des contraintes et choix de la collectivité, etc...

D'un point de vue éthique, cet apport est particulièrement utile: plutôt que de tout ramener à des considérations d'ordre monétaire, il permet d'inclure cet aspect parmi d'autres, de mieux prendre en compte des points de vue différents, d'en mesurer le poids relatif et donc de s'orienter vers une décision davantage éclairée, et donc, responsable.

L'élaboration d'outils d'aide à la décision médicale, à laquelle collaborent actuellement des économistes (qu'il s'agisse de la constitution d'arbres de décision, d'analyses coût-efficacité ou coût-bénéfice, ou d'évaluation des appareillages, des procédures ou des traitements) devrait connaître des développements importants et féconds à l'avenir [15], [16]

Certes, beaucoup de travail reste à faire: tout comme le médecin n'a pas trouvé l'élixirmiracle, l'économiste n'a pas encore découvert la formule magique de l'analyse coût-efficacité parfaite [3]. Sans doute faut-il veiller à rendre de plus en plus opérationnelle cette démarche, mais l'esprit et le fondement pédagogique qui la sous-tendent, ainsi que l'interdisciplinarité qu'elle exige, constituent déjà des avancées nécessaires [1] et, à terme, fécondes.

\section{Rôle des systèmes de santé dans le degré d'intégration des contraintes économiques dans la décision médicale}

La deuxième partie de notre commentaire naît d'une constatation et d'une question. D'une constatation tout d'abord: c'est aux Etats-Unis, aux alentours des années 1970, que l'élaboration des modèles de décision médicale s'est fortement développée dans la ligne des travaux de prise de décision en incertitude effectués par VON NEUMANN et MORGENSTERN en 1947. La France suit la même démarche, mais avec un décalage d'une dizaine d'années: après une période de relative abondance des moyens durant laquelle l'insuffisance de régulation économique du système de soins a entraîné une croissance rapide des dépenses de santé (de 6 points supérieure à celle du PIB sur la période 1950-1980) [18], [19], on a assisté, au début de cette décennie, à une remise en cause des modes de financement du système de soins fran çais [20] envisageant notamment la possibilité d'un grand recours à des modalités privées de financement. Ce n'est que vers le milieu des années 1980 que se généralisent les approches coûts-bénéfices, à la demande notamment des hôpitaux et des laboratoires hospitaliers (soumis à partir de 1984-1985 au financement plus contraignant par dotation annuelle globale) ou de la Sécurité Sociale. Ce resserrement des contraintes oblige à se réinterroger sur la rationalité des choix [1].

Cette double constatation entraîne une question: n'y-a-t-il pas des systèmes de soins dont l'organisation favorise l'intégration des contraintes économiques dans la décision 
médicale? Pour répondre à cette question, nous comparerons très brièvement les systèmes des USA, du Royaume-Uni et de la France [4].

L'analyse des systèmes américain, anglais et français montre que, dans ces deux types d'organisation des soins de santé, les acteurs sont incités, mais de façon différente, à introduire le critère économique dans leur choix en matière de soins.

- Aux Etats-Unis, au moins quatre catégories de partenaires peuvent se sentir concernés par un meilleur usage des ressources consacrées à la santé et donc par un plus grand recours aux méthodes de rationalisation des choix et des décisions en incertitude [1]:

- les assureurs doivent proposer une couverture sociale qui rende maximum l'impact de l'assurance sur la santé, compte tenu d'un niveau de prime donné; ce niveau constitue ainsi le régulateur sur le marché américain de l'assurance-santé;- ensuite, les médecins et les administratifs hospitaliers, auxquels est imposé un système de remboursement forfaitaire des dépenses à l'hôpital, sont incités à déterminer entre eux la meilleure allocation bénéfices/ coûts des ressources hospitalières;

- en troisième lieu, les H.M.O., système d'assurance regroupant les assureurs, les assurés et le corps médical dans des organisations ayant pour objectif d'assurer la santé de leurs membres ("Health Maintenance Organization") au meilleur rapport qualité/prix, pourrait-on dire, ont tout intérêt à allouer efficacement un budget fixé a priori;

- enfin, les patients, dans le cadre des assurances privées ou des réseaux de santé qui leur sont proposés, doivent prendre les meilleures décisions d'allocation de leur budget, notamment à l'égard du poste SANTÉ.

Une exception mérite d'être soulignée, celle du système du Medicare (pour les personnes agées) ou du Medicaid (pour les plus pauvres), dont le financement se rapproche fortement de celui opéré par notre système de Sécurité Sociale et qui a connu une croissance accélérée et bien plus vive que celle observée dans d'autres secteurs de la santé aux Etats-Unis; à la suite de l'adoption de Medicare et Medicaid, les dépenses de santé financées par les Etats ou le gouvernement fédéral sont passées de 20 à $40 \%$ de l'ensemble des dépenses de santé. Ces dernières, calculées par habitant, ont, par ailleurs, doublé entre 1965 et 1978 [23].

Ainsi, mise à part l'exception rapidement évoquée à l'instant, les systèmes de régulation et/ou d'incitation existant aux Etats-Unis (système d'assurance privée, prime d'assurance en milieu concurrentiel; remboursement forfaitaire des soins à l'hôpital; prime forfaitaire d'assurance pour les réseaux de santé - H.M.O., P.P.O. ${ }^{2}$-) engagent tous les acteurs à la recherche d'une meilleure allocation bénéfices/coûts des ressources consacrées à la santé.

- Dans le système britannique du National Health Service, il existe un autre élément de régulation du système et de ses dépenses, qui est, cette fois, l'offre de soins, déterminée de façon autoritaire par les Pouvoirs Publics. A la régulation par les prix (et donc la concurrence), il faut ajouter un autre moyen de contrôle d'un système de santé et de ses évolutions, qui est le contrôle public.

- Dans le cas français, aucune de ces deux régulations n'est appliquée à l'ensemble du système. En effet, ni le patient (qui est consommateur sans être décideur ou payeur), ni le médecin, qui décide de sa prescription, sans contraintes financières importantes, ni

\footnotetext{
${ }^{2}$ Preferred-Provider Organizations.
} 
l'Administration ou la Sécurité Sociale, qui ne sont que les bailleurs des fonds distribués a posteriori et qui n'ont que très peu de contrôle sur le niveau et l'efficacité des dépenses, ne sont véritablement incités à déterminer les choix les plus rationnels et les véritables priorités dans le domaine de la santé. Le système de soins, libéral du côté de l'offre et de la demande, socialisé du côté financement, apparaît alors peu ou mal régulé.

A cet égard, l'instauration récente du budget global à l'hôpital lève en partie cette carence et contraint fortement les établissements à rechercher une meilleure gestion des ressources qui leur sont attribuées. Mais, dans un contexte de budgets en croissance très limitée d'une année à l'autre (parfois inférieure à la hausse générale des prix), cette contrainte est douloureusement ressentie à court terme et semble arriver ou trop tard ou trop tôt [21].

Plus largement, commencent à être étudiées des tentatives de restauration d'un minimum de mécanismes incitatifs susceptibles de réintroduire une forme de contrôle par le marché: la formule des réseaux de soins coordonnés va dans ce sens [24].

Ainsi, le système américain de soins de santé, de nature libérale tant dans son financement que dans sa production, a été contraint, beaucoup plus vite que le système français libéral dans la distribution des soins mais socialisé dans leur financement - d'instaurer les éléments de sa régulation et les facteurs d'incitation obligeant les acteurs à tenir compte des contraintes économiques en vigueur. Ceci ne va pas sans poser de questions: il convient maintenant de s'interroger, non seulement sur la délivrance et le financement des soins, mais également sur les résultats des actions et décisions en termes de santé. A cet égard, les questions relatives à l'accès aux soins et à la qualité des soins soulèvent de plus en plus d'interrogations, dans le système américain notamment [22].

En définitive, du point de vue économique et du point de vue éthique, la plus grande prise en compte des contraintes économiques dans la prise de décision médicale [17], qu'elle soit favorisée par l'organisation du système de santé (comme aux USA ou en Grande-Bretagne) ou qu'elle soit exigée par le resserrement des contraintes (comme en France), oblige à réinterroger la pratique existante. De ce point de vue, les analyses coût-efficacité et celles mises en œuvre dans le domaine de l'aide à la décision médicale soulignent bien la nécessité de réintroduire des critères et des mécanismes économiques, soit dans le processus de choix et d'établissement de priorités (en ce qui concerne les critères), soit dans les éléments de la politique sanitaire (pour ce qui est des mécanismes).

Un Président du Conseil français, Georges CLÉMENCEAU, disait que la guerre est une affaire trop importante pour être laissée aux militaires... Prolongeant cette boutade, on pourrait ajouter que l'économie est trop essentielle pour être abandonnée aux seuls économistes et que la santé est trop précieuse pour être confiée aux seuls médecins!

Sans doute serions-nous d'accord pour dire que médecins et économistes, avec d'autres, devront s'atteler, ensemble, à la tâche suivante: déterminer la meilleure façon de procurer à tous la plus grande qualité de santé au moindre coût. 


\section{BIBLIOGRAPHIE}

[1] WEINSTEIN, M.: "Risky choices in medical decision-making: a survey", presentation at the Ninth Annual Lecture of the Geneva Association, Faculté Universitaire Catholique de Mons, Belgium, 8 November 1985.

[2] LOEWY, E.H.: "Cost should not be a factor in medical care", letter from the New England of Medicine, Vol. 302, No. 12.

[3] WILLIAMS, A.: "Medical ethics, health service efficiency and clinical freedom", Nuffield/York Portfolios, Folio 2, 50 p.

[4] SAILLY, J.C., LEBRUN, T.: "La santé dans un monde en crise", document ronéoté, CRESGE, Lille, automne $1985,35 \mathrm{p}$.

[5] SAILLY, J.C., LEBRUN, T: "Système de production de soins et dèpenses de santé en France: vers des éléments de régulation?", Journal d'Economie Médicale, T.2, 1984, pp. 125-146.

[6] LEIGHTON READ, J., QUINN, R.J., BERWICK, D.M., FINEBERG, H.V., WEINSTEIN, M.C.: "Preferences for health outcomes - comparison of assessment methods", Medical Decision-Making, Vol. 4, No. 3, 1984, pp. 315-329.

[7] WEINSTEIN, M., STASON, W.: "Cost-effectiveness of coronary artery bypass surgery", Circulation 66, Suppl. III, 1982, pp. 56-65.

[8] ERAKER S., SOX, H.: "Assessment of patients' preferences for therapeutic outcomes", Medical Decision-Making, Vol. 1, No. 1, 1981.

[9] SUlMAN, C., EECKHOUDT, L., DEMAILlE, J.C., CARPENTIER, P., VENIN, P., LEBRUN, T. "Evaluation des coûts et bénéfices du traitement des métastases osseuses: la méthode des loteries", Travaux CRESGE, Centre Oscar Lambret, Lille, 1984.

[10] MIYAMOTO, J., ERAKER, S.: "Parameter estimates for a qaly utility model", Medical DecisionMaking, in Press.

[11] LEBRUN, T., SAILLY, J.C., EECKHOUDT, L.: "Selection of disgnostic tests: a comparison between an information theory and a decision-making approach. The illustrative case of coronary artery disease”. Health Economists' Study Group, London, January 1985.

[12] SAILLY, J.C., LEBRUN, T., EECKHOUDT, L., MELIN, J., VANBUTSELE, R., ROBERT, A., BROHET, C., DETRY, J.M.: "Les apports de la théorie de l'information et de l'approche en termes d'aide à la décision médicale dans le choix d'examens complémentaires: le cas des maladies coronariennes", communication au Colloque de l'Association Française de Sciences Economiques, Paris, septembre 1985, et au Colloque Franco-Allemand CNRS d'Arc-et-Senans, octobre 1985.

[13] SAILlY, J.C., LEBRUN, T., VINCENT, P., EECKHOUDT, L., IZARD, D., LECLERC, H., ARBON, G. et HASSOUN, A.: "The medical and economic consequences of automation in bacteriology", a case study in a French University Hospital, Social Science and Medicine, Vol. 21, No. 10, pp. 1163-1166, 1985.

[14] FALISE, M.: "Une pratique chrétienne de l'économie", Le Centurion, 1985, 199 p.

[15] NCHSR and HCTA: “"Health services research on primary care”, Program Note, U.S. Department of Health and Human Services, Public Health Service, may 1985, 8 p.

[16] NEUHAUSER, D.: "Stimulating cost-effective behaviour in hospitals", 3rd Symposium Health and Economics, Antwerp, September 1985, pp. 29-30.

[17] LEBRUN, T., SAILLY, J.C., LEFEVRE, C.: "Contraintes économiques et choix thérapeutiques", Médecine de l'Homme, no 154, novembre-décembre 1984, pp. 14-23.

[18] LAMBERT, D.C.: "La croissance des dépenses de santé peut-elle durablement excéder celle des ressources de la nation?", Lyon, 1975.

[19] SAMAILLE, J.: "La santé est-elle au-dessus de nos moyens?, Savoir, URCEAS, no 358, février 1983, $4 \mathrm{p}$. 
[20] LAMBERT, D.C.: "Faudra-t-il étendre le recours à l'assurance privée pour maîtriser durablement les dépenses de santé en France?", Journal d'Economie Médicale, T. 1, no 21, 1983, pp. 63-76 et T. 1, no 3, 1983, pp. 135-150.

[21] SAILLY, J.C., LEBRUN, T.: "Le budget global à l'hôpital", éditeur CRESGE, Institut Pasteur, IESEG, Lille, Mai 1985, 327 p. + annexes.

[22] ENTHOVEN, A.C.: "The U.S. health care economy: from guild to market in ten years", 3rd Symposium Health and Economics, Antwerp, September 1985, pp. 35-38.

[23] RODWIN, V.G.: "La santé et le paradoxe de la politique Reagan”, Prospective et Santé, pp. 63-70.

[24] GIRAUD, P. et LAUNOIS, R.J.: “Les réseaux de soins, médecine de demain”, Economica, 1985, $154 \mathrm{p}$. 